\title{
Aplicação da técnica de eye tracking em pesquisas envolvendo a tomada de decisão: uma análise bibliométrica dos estudos publicados entre 2009 e 2019
}

\author{
Application of the eye tracking technique in research involving decision making: a \\ bibliometric analysis of studies published between 2009 and 2019
}

Eliete dos Reis Lehnhart Doutora em Administração pela Universidade Federal de Santa Maria - UFSM, Brasil. Professora Adjunta da Universidade Federal de Santa Maria - UFSM, Brasil. https://orcid.org/0000-0001-6676-8547

E-mail: elietedosreis@gmail.com

Daiane Lindner Radons Doutoranda em Administração pela Universidade Federal de Santa Maria - UFSM, Brasil. https://orcid.org/0000-0003-3545-0741

E-mail: daialindner@gmail.com

Rafaela Dutra Tagliapietra Mestra em Administração pela Universidade Federal de Santa Maria - UFSM, Brasil. https://orcid.org/0000-0002-5436-5705

E-mail: rafatagliapietra@gmail.com

Julia Tontini

Doutoranda em Administração pela Universidade Federal de Santa Maria - UFSM, Brasil. https://orcid.org/0000-0001-8509-8665

E-mail: ju-tontini@hotmail.com

\section{Resumo}

Este trabalho investiga a produção de estudos que envolvem o uso de eye tracking em pesquisas sobre tomada de decisão, por meio de uma análise bibliométrica de trabalhos publicados na Web of Science, no período de 2009 a 2019. Como objetivos específicos, pretende-se: i) realizar uma análise bibliométrica dos estudos que utilizaram a técnica de eye tracking em pesquisas sobre tomada de decisão nas áreas de administração; ii) identificar as estratégias de pesquisa dos artigos selecionados de acordo com os parâmetros do índice $h$. Para tanto, analisaramse as estratégias de pesquisa empregadas pelos 18 estudos incluídos nos parâmetros do índice $h$. Os resultados evidenciaram que 2018 foi o ano que apresentou o maior número de trabalhos, que as categorias negócios e gestão lideram o ranking das publicações e que todos os trabalhos estão vinculados à área de Economia e Negócios, sendo alguns associados a outras áreas. Sobre a autoria desses estudos, observou-se uma distribuição homogênea entre o número de artigos por autor com um predomínio norte-americano nas publicações sobre o tema. No que se refere às estratégias de pesquisa, a maioria corresponde aos estudos quantitativos, explicativos, fazendo uso da abordagem experimental, tendo utilizado para a coleta de dados o eye tracking.

Palavras-chave: Bibliometria. Eye tracking. Decisão. Estratégias metodológicas. Web of Science.

\begin{abstract}
This work investigates the production of studies that involve the use of eye tracking in research on decision making, through a bibliometric analysis of works published in the Web of Science, in the period from 2009 to 2019. The specific objectives are to: i) Conduct a bibliometric analysis of studies that used the eye tracking technique in research on decision making in the areas of administration; ii) Identify the search strategies for the selected articles according to the parameters of the $\mathrm{h}$ index. Therefore, the research strategies employed by the 18 studies included in the parameters of the $h$ index were analyzed. The results showed that 2018 was the year that presented the largest number of works, that the business and management categories lead the ranking of publications and that all works are linked to the area of Economics and Business, some of which are associated with other areas. Regarding the authorship of these studies, there was a homogeneous distribution among the number of articles per author with a North American predominance in publications on the subject. With regard to research strategies, most correspond to quantitative, explanatory studies, using the experimental approach, having used eye tracking for data collection.
\end{abstract}

Keywords: Bibliometry. Eye tracking. Decision. Methodological strategies. Web of Science.

InCID: R. Ci. Inf. e Doc., Ribeirão Preto, v. 12, n. 1, p. 41-59, mar./ago. 2021.

DOI: 10.11606/issn.2178-2075.v12i1p41-59 


\section{Introdução}

A forma diversificada de pensamento dos indivíduos, no entendimento de Glynn et al. (2017), determina como estes decidem, agem e cooperam entre si. Desse modo, buscar entender como a mente humana funciona é primordial para compreender o processo de tomada de decisão (BENDER, 2019). Um método eficiente considerado para explicar tais questões é a técnica eye tracking (MEIßNER; MUSALEM; HUBER, 2016).

O eye tracking ou rastreamento ocular é uma técnica que permite verificar onde um indivíduo está olhando a partir do movimento dos seus olhos, gravar e ordenar sua visualização, além de considerar a duração (tempo) da fixação em cada ponto visualizado (MELCHER, 2012). Casalinho (2016) corrobora salientando que o eye tracking possibilita a compreensão do processo cognitivo das pessoas, ao utilizar o movimento dos olhos como parâmetro.

O rastreamento ocular permite obter informações mais realísticas, pois é possível compreender o processo de decisão através da atenção visual de um indivíduo (MEIßNER; DECKER, 2010), com isso, estudos que contemplam o uso do eye tracking na tomada de decisão vem se desenvolvendo em diferentes perspectivas (HOLMQVIST et al., 2011; MEIßNER; MUSALEM; HUBER, 2016). Neste âmbito, verifica-se a importância de compreender como são desenvolvidos os estudos sobre o uso do eye tracking na tomada de decisão, tendo em vista que estudos bibliométricos são característicos para alcançar esse objetivo, ao analisar a qualidade e o desenvolvimento do conhecimento e produção científica de um determinado assunto (PIMENTA et al., 2017). Dessa forma, esta investigação busca explorar a utilização de uma técnica de rastreamento ocular na compreensão do processo decisório a partir do seguinte problema de pesquisa "Quais as características da produção científica sobre eye tracking em pesquisas de tomada de decisão no período de 2009 a 2019?".

Nesse sentido, este artigo investiga a produção de estudos que envolvem o uso de eye tracking em pesquisas sobre tomada de decisão, por meio de uma análise bibliométrica de trabalhos publicados na Web of Science, no período de 2009 a 2019. Como objetivos específicos, pretende-se: i) realizar uma análise bibliométrica dos estudos que utilizaram a técnica de eye tracking em pesquisas sobre tomada de decisão nas áreas de administração; ii) identificar as estratégias de pesquisa dos artigos selecionados de acordo com os parâmetros do índice $h$. 
Este trabalho apresenta contribuições para o campo teórico e prático. Ao apresentar um panorama sobre os estudos envolvendo o uso do eye tracking na tomada de decisão, relação esta, ainda não explorada na literatura, possibilita-se avançar na compreensão científica sobre o assunto, identificando estudos que utilizaram o registro dos movimentos dos olhos para elucidar aspectos relevantes no processo decisório. Além disso, são fornecidos subsídios aos pesquisadores para que realizem suas escolhas epistemológicas e metodológicas, acompanhando a evolução e as tendências das publicações. A partir desta investigação, promove-se o desenvolvimento do campo teórico científico sobre o tema, apontando lacunas para novas contribuições práticas, em especial, com o uso da ferramenta de rastreamento ocular.

Na primeira e na segunda parte deste artigo, encontra-se a introdução e o referencial teórico, destacando os principais aspectos sobre a tomada de decisão, perspectiva Neuro-IS e eye tracking. Na terceira parte detalha-se o método do estudo seguido do resultado. Por último, nas considerações finais são apresentados os principais achados da pesquisa, as limitações e as implicações para a teoria e os caminhos para investigações futuras.

\section{Referencial teórico}

\subsection{Tomada de decisão}

Diariamente, as pessoas decidem sobre seus cursos de ação, sendo influenciadas por variáveis comportamentais e ambientais. Glynn et al. (2017) ressaltam que o reconhecimento de tais influências é essencial, pois elas afetam a produção e o uso de informações em várias áreas da sociedade. Nesta perspectiva, a tomada de decisão é um tema amplo que marca presença na agenda de pesquisas desde meados do século dezoito (EDWARDS, 1954).

Conforme Gomes (2007), a tomada de decisão é entendida como um processo de escolha entre diversas alternativas disponíveis, processo este que é influenciado pela inteligência, características psicológicas do decisor e pelo ambiente onde este está inserido. Para Pérez, Cabrerizo e Herrera-Viedma (2010), o processo decisório consiste em derivar o melhor resultado dentre as alternativas possíveis e está presente na maioria das tarefas humanas.

De acordo com o modelo racional da decisão, os indivíduos possuem informações perfeitas e são capazes de avaliar racionalmente cada alternativa para tomar uma decisão 
totalmente imparcial e condizente (PEREIRA; LÖBLER; SIMONETTO, 2010). Tal modelo não considera as limitações e as subjetividades da mente humana e, conforme enfatizam Bazerman e Moore (2010), baseia-se em um conjunto de premissas que determinam como uma decisão deve ser tomada e não descrevem como uma decisão é tomada.

Embora que os decisores buscam ser racionais por meio de seus comportamentos, eles estão submetidos às limitações no processo. Segundo Simon (1955), o julgamento individual fica limitado pela racionalidade e o processo decisório poderia ser mais bem compreendido a partir da explicação de decisões reais em vez de analisar apenas a decisão prescritiva. Para o autor, o processamento de informações exigido na tomada de decisão racional, extrapolam as capacidades cognitivas dos indivíduos, dessa forma, é necessário o uso de heurísticas, isto significa utilizar de estratégias simplificadoras ou regras práticas que possam contribuir no processo de decisão (BAZERMAN; MOORE, 2010), reduzindo a carga cognitiva e acelerando a tomada de decisão. Como resultado desse processo, os decisores buscam decisões satisfatórias ao contrário de decisões ótimas.

Para Glynn et al. (2017), a mente humana afeta as decisões individuais e sociais, inclusive as relacionadas à ciência, à política e às ações de gerenciamento. Os autores sustentam que as decisões geralmente são reativas às experiências mais recentes do indivíduo, ainda que associadas às lições do passado por meio de adaptações genéticas e culturais. Dessa forma, se verifica a importância da compreensão dos aspectos que influenciam o processo de decisão, sobretudo, aqueles inerentes ao ser humano, como o estudo da mente e da cognição.

\subsection{Perspectiva Neuro-IS}

De acordo com Bender (2019), para entender como os indivíduos tomam suas decisões é necessário compreender como funciona seu raciocínio. Nesse sentido, busca-se, por meio da mensuração da atividade cerebral implícito aos diferentes processos de tomada de decisão (RIEDL; DAVIS; HEVNER, 2014), utilizar a abordagem Neuro-IS para aprimorar o conhecimento da mente humana na área de Sistemas de Informação (SI).

Na concepção de Velho et al. (2016), a perspectiva Neuro-IS examina os efeitos neurofisiológicos relacionados à concepção, uso e impacto de diferentes tecnologias da informação, propiciando uma nova abordagem para análise de SIs. Assim, pesquisadores da área encontraram nas técnicas de Neurociência a maneira adequada para tal investigação 
(BUETTNER, 2015; VELHO et al., 2016). Um exemplo é o eye tracking, ou rastreamento ocular, que explora o comportamento dos olhos do indivíduo em diferentes espaços, acompanhando e realizando a gravação de todo o movimento ocular (BARRETO, 2013; RODAS; MARCOS; VIDOTTI, 2014).

Segundo Zamani, Abas e Amin (2016), as métricas originadas do mapeamento dos movimentos oculares, a partir da técnica eye tracking, podem ser utilizadas para i) analisar o design de determinado software, interações com programas e tarefas de pesquisa na web; ii) medir a atividade neural dos indivíduos enquanto percebem e processam determinado estímulo visual; iii) avaliar a usabilidade computacional, comparando a eficácia de diferentes interfaces; iv) compreender a interação humano-computador e a interação cérebro-computador; v) mapear a tecnologia assistiva, e-learning e realidade virtual e aumentada.

Ao analisar o estudo da tomada de decisão, é importante destacar a investigação de aspectos comportamentais e cognitivos pertinentes às escolhas no ambiente virtual. Com a adoção de técnicas de Neurociências é possível aliar a análise dos dados coletados através de métodos tradicionais com métricas proporcionadas pelo rastreamento ocular (ROULLET; DROULERS, 2010).

Sob esse prisma, a análise da tomada de decisão com base no rastreamento ocular pressupõe a hipótese strong eye-mind. Tal premissa enfatiza que o comportamento desses movimentos se relaciona com a atenção direcionada pelo indivíduo durante determinada tarefa. Assim, considera-se a região que o indivíduo visualiza como um indicador do seu pensamento (BARRETO, 2013), proporcionando compreender, em parte, o processo cognitivo do usuário (RODAS; MARCOS; VIDOTTI, 2014). Nesse sentido, Chae e Lee (2013) apontam que para entender o processo de tomada de decisão humana com maior precisão é necessário considerar os processos perceptuais, emocionais e cognitivos do indivíduo durante o processo de escolha (CHAE; LEE, 2013). Dessa forma, em seguida, menciona-se conceitos sobre o rastreamento ocular.

\subsection{Eye tracking}

No ano de 1965, Hess ficou intrigado com a dilatação da pupila em determinadas situações cotidianas, o que fez com que ele realizasse diferentes experimentos para compreender seu significado. Ele comprovou que as pupilas indicam um esforço mental de um indivíduo em reação a diferentes níveis de processamento da informação, demonstrando que, 
quanto maior o nível de processamento da informação, maior é a dilatação das pupilas. Corroborando com esses achados, Yarbus (1967) inseriu a técnica de rastreamento ocular (eye tracking) com o objetivo de compreender diferentes problemas. $\mathrm{O}$ autor concluiu que, quando um objeto se move, os olhos se movimentam em sua direção, gerando uma imagem na retina para identificar esse objeto.

A partir desses estudos, a técnica eye tracking permitiu a compreensão do comportamento cognitivo dos indivíduos por meio do movimento dos olhos (CASALINHO, 2016). Just e Carpenter (1980) expuseram a hipótese do olho-mente, considerando que os olhos se mantêm fixados em um determinado objeto ou palavras, ao mesmo tempo que sua mente está processando essa imagem, comprovando uma forte relação entre a cognição e os movimentos oculares.

Existem diferentes maneiras para analisar o rastreamento ocular em relação a compreensão do processamento cognitivo de um indivíduo, sendo que as técnicas mais conhecidas e básicas são as sacadas e as fixações (RAYNER, 1998; BARRETO, 2013). As sacadas referem-se aos movimentos rápidos dos olhos, com uma velocidade de 500 milissegundos (ms), geralmente ocorrem quando uma pessoa está olhando para objetos ou realizando uma leitura, por exemplo. Já as fixações, correspondem ao momento em que os olhos permanecem fixos em uma área, possuindo uma velocidade de 200 a 300 ms. As fixações ocorrem entre as sacadas, ou seja, quando um indivíduo está movimentando os olhos de um local para outro, em determinado momento eles param imóveis, para visualizar um objetivo, ocorrendo as fixações (RAYNER, 1998).

Diante disso, investigações com o uso do eye tracking estão se desenvolvendo em diferentes áreas de pesquisa (HOLMQVIST et al., 2011). Uma dessas áreas é a tomada de decisão. Como exemplo, pode-se pensar na concepção de Meißner, Musalem e Huber (2016) segundo os quais, os movimentos oculares forneceram achados importantes sobre os processos subjacentes da escolha de um produto por parte do consumidor. Para os autores, com a observação dos movimentos oculares é possível obter uma compreensão mais clara sobre os processos que os indivíduos utilizam para realizar um exercício, envolvendo a escolha dentre alternativas em uma tarefa. Com isso, investigar a utilização do eye tracking em pesquisas sobre tomada de decisão conduz a um entendimento atual deste campo de forma mais abrangente. 


\section{Método}

A investigação da produção de estudos que envolvem o uso de eye tracking em pesquisas sobre tomada de decisão consistiu em um estudo bibliométrico, de natureza quantitativa e descritiva. De acordo com Wolfram (2017) a abordagem bibliométrica é realizada para entender o desenvolvimento da produção científica, ao utilizar uma quantidade de dados em determinado período, a partir de recursos estatísticos e matemáticos

A coleta de dados foi realizada por meio de desk research, na qual, utilizaram-se os termos decis* e eye track* na ferramenta de busca "tópico" que abrange informações do tema contidas no "título", "resumo" e "palavras-chave" dos trabalhos disponíveis na plataforma Web of Science (WOS), base de dados que fornece acesso a referências e resumos em todas as áreas do conhecimento. Além disso, fez-se uso do filtro somente artigos nas categorias Business, Business Finance e Management para o período de 2009 a 2019, com o intuito de abranger estudos relacionados à Administração. Destaca-se que a categoria Business Finance contempla trabalhos de negócios e de finanças, perfazendo a área de interesse.

A primeira busca resultou em 1.175 artigos, desses foram eliminados 1.100 por não estarem relacionados às categorias acima mencionadas. Posteriormente a esse filtro, resultaram 75 trabalhos para a análise bibliométrica (segunda etapa). Para análise das estratégias de pesquisa (terceira etapa), optou-se por realizá-la de acordo com os parâmetros do índice $h$. Dessa forma, 18 artigos que estavam incluídos nesse parâmetro, de quantificação da produtividade e impacto de pesquisas, foram selecionados para tal análise.

O índice $h$ é obtido a partir do relatório de citações na Web of Science que compreende a classificação das publicações em ordem decrescente de citações. Conforme Web of Science (2020), um índice $h$ representa que existem $h$ artigos em que cada um foi citado, no mínimo, $h$ vezes. No caso desta pesquisa, há 18 trabalhos que foram citados, pelo menos, 18 vezes. Entre as publicações selecionadas, o primeiro artigo da lista possui 94 citações e o último conta com 19 citações. Preferiu-se por analisar as estratégias de pesquisa dos artigos mais citados, com base no índice $h$, considerando o impacto desses trabalhos para a área de interesse e visando apresentar um panorama mais acurado dos artigos em termos metodológicos, os quais poderão contribuir com o desenvolvimento de novas pesquisas a partir de lacunas identificadas. A Figura 1 apresenta as etapas do estudo bibliométrico. 
Figura 1 - Etapas do estudo bibliométrico

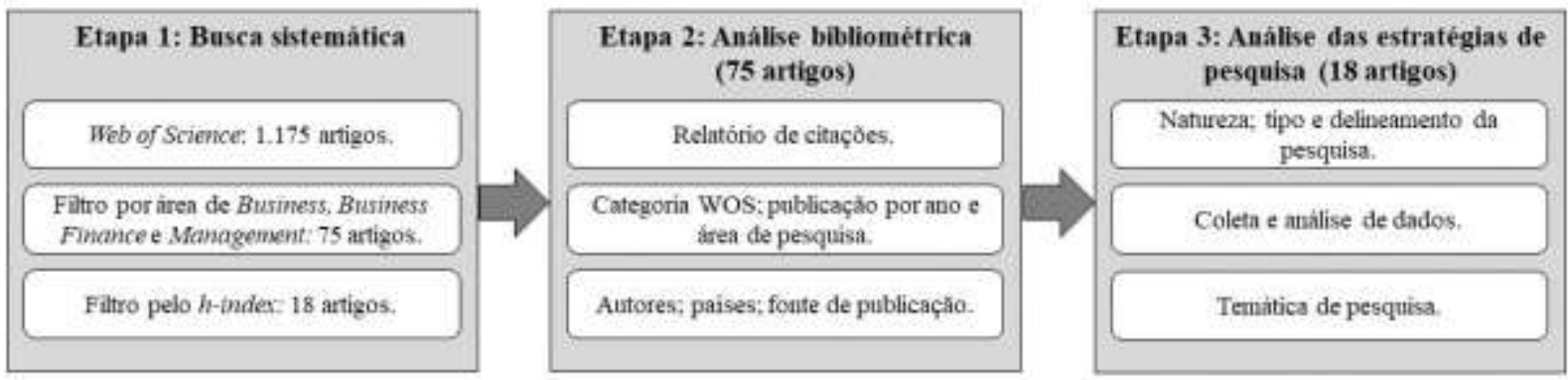

Fonte: elaborada pelos autores.

Os resultados das buscas foram exportados para a ferramenta Microsoft Excel, o qual serviu para análises e construção de gráficos apresentados neste trabalho. Dessa forma, inicialmente as análises foram referentes a categoria da WOS, ano de publicação, áreas de pesquisa, autores, países e fonte de publicação. Em seguida conduziu-se a análise das estratégias de pesquisa utilizadas nos 18 estudos de acordo com: (i) natureza da pesquisa, (ii) tipo de pesquisa, (iii) delineamento da pesquisa, (iv) coleta e análise dos dados e (v) temática da pesquisa. Para tanto, procedeu-se uma leitura sistemática dos resumos e, na sequência, a leitura do método dos estudos. Em determinados casos, houve a leitura integral dos textos, buscando uma maior compreensão das estratégicas metodológicas adotadas. Os resultados são apresentados na próxima seção.

\section{Análise e discussão dos resultados}

Os resultados são apresentados em duas seções: i) análise bibliométrica dos 75 trabalhos; e, ii) análise das estratégias de pesquisa de 18 artigos, selecionados de acordo com os parâmetros do índice $h$.

\subsection{Análise bibliométrica}

O total de 75 artigos estão divididos em 15 categorias da Web of Science (Tabela 1), sendo que alguns trabalhos são considerados em mais de uma categoria. Destaca-se que negócios e gestão lideram o ranking, perfazendo mais de $64 \%$ do total de publicações, corroborando as categorias selecionadas na primeira etapa do estudo: Business, Business Finance e Management. 
Tabela 1 - Categorias Web of Science

\begin{tabular}{|c|c|c|}
\hline Categoria & $\begin{array}{l}\text { Total de } \\
\text { trabalhos }\end{array}$ & $\%$ do total \\
\hline Negócios (Business) & 53 & 44,5 \\
\hline Gestão (Management) & 25 & 21,0 \\
\hline Negócios, Finanças (Business Finance) & 8 & 6,7 \\
\hline Psicologia Aplicada (Psychology Applied) & 7 & 5,9 \\
\hline $\begin{array}{l}\text { Sistemas de Informação da Ciência da Computação (Computer Science } \\
\text { Information Systems) }\end{array}$ & 5 & 4,2 \\
\hline Economia (Economics) & 4 & 3,4 \\
\hline Comunicação (Communication) & 3 & 2,5 \\
\hline Ciência da Informação Biblioteconomia (Information Science Library Science) & 3 & 2,5 \\
\hline Psicologia Social (Psychology Social) & 3 & 2,5 \\
\hline $\begin{array}{l}\text { Ciência de Gestão na Pesquisa Operacional (Operations Research Management } \\
\text { Science) }\end{array}$ & 2 & 1,7 \\
\hline Telecomunicações (Telecommunications) & 2 & 1,7 \\
\hline $\begin{array}{l}\text { Ciência do Computador Inteligência Artificial (Computer Science Artificial } \\
\text { Intelligence) }\end{array}$ & 1 & 0,8 \\
\hline $\begin{array}{l}\text { Aplicações Interdisciplinares de Ciência Computacional (Computer Science } \\
\text { Interdisciplinary Applications) }\end{array}$ & 1 & 0,8 \\
\hline Engenharia e Fabricação (Engineering Manufacturing) & 1 & 0,8 \\
\hline Ergonomia (Ergonomics) & 1 & 0,8 \\
\hline Total & $119 *$ & 100 \\
\hline
\end{tabular}

*As categorias podem ser múltiplas, superior ao total de artigos.

Fonte: elaborada pelos autores.

Na Figura 2, estão apresentadas as áreas de pesquisa dos trabalhos, considerando o ano de publicação. Conforme demonstrado, todos os trabalhos estão vinculados à área de Economia e Negócios e alguns artigos estão associados à outras áreas, como a Psicologia, que possui 7 publicações; Ciência da Computação, com 6 trabalhos; Comunicação e Ciência da Informação e Biblioteconomia, ambas com 3 trabalhos.

Quanto aos anos de publicação, 2018 apresenta o maior número de trabalhos, 14, seguido de $2019(\mathrm{~N}=13), 2016(\mathrm{~N}=12)$ e $2015(\mathrm{~N}=11)$. Os demais anos possuem 6 ou menos trabalhos. Evidencia-se, assim, que a temática do estudo é atual e possui potencial de investigação, já que os últimos dois anos são responsáveis por 36\% das publicações.

Ainda sobre a evolução das publicações, vale destacar que, em uma busca rápida na base de dados Web of Science (2021) com o termo "eye tracking", verifica-se que, ao analisar os resultados dessas publicações, o número de trabalhos sobre a ferramenta aumentou entre o período mais recente, 2018 a 2020. Dessa forma, compreende-se que o tema em questão está em ascensão e vai ao encontro com os achados nesta investigação, em que o uso de eye tracking em pesquisas sobre tomada de decisão possui seu desenvolvimento nos últimos anos. 
Figura 2 - Áreas de pesquisa por ano de publicação

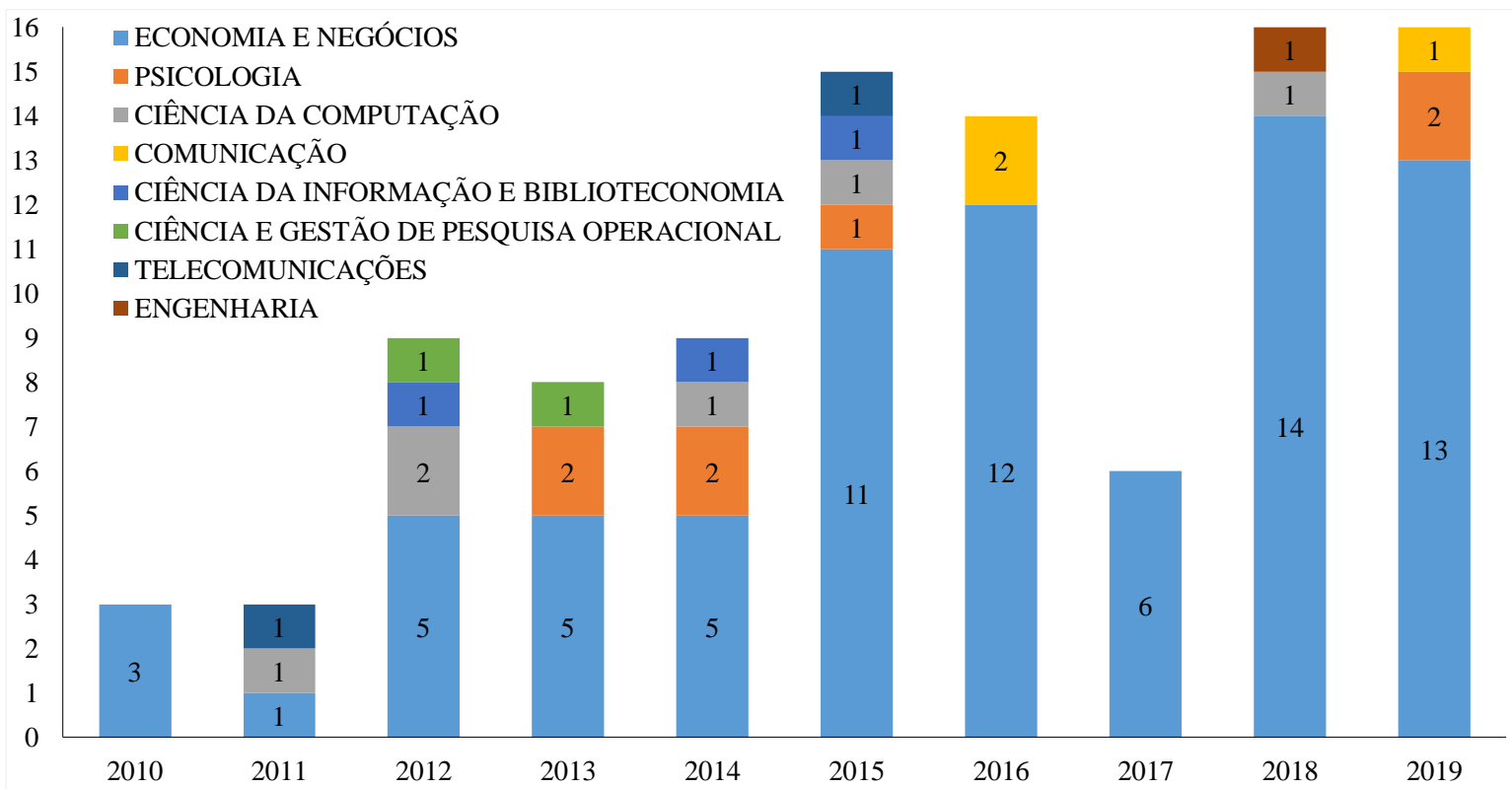

Fonte: elaborada pelos autores.

Em relação aos autores, nota-se uma distribuição homogênea entre o número de publicações, uma vez que os pesquisadores publicaram um ou dois artigos. Na Tabela 2, são apresentados os autores que possuem dois trabalhos e seus respectivos países. Destaca-se que os autores que possuem publicações em coautoria foram agrupados.

Tabela 2 - Autores, número de trabalhos e países

\begin{tabular}{l|c|c}
\hline Autor(es) & $\mathbf{N}^{\circ}$ trabalhos & País \\
\hline Behe, B. K.; Huddleston, P.; Minahan. S. & 2 & Estados Unidos \\
\hline Bercik, J.; Viragh, R. & 2 & Eslováquia \\
\hline Clement, J. & 2 & Dinamarca \\
\hline De Jong, M. G.; Toubia, O.; Yang, L. & 2 & França \\
\hline Gustafsson, A.; Otterbring, T.; Wastlund, E. & 2 & Suécia \\
\hline Huang, Y. F.; Kuo, F. Y. & 2 & Taiwan \\
\hline Kahn, B. E. & 2 & Estados Unidos \\
\hline Meissner, M. & 2 & Austrália \\
\hline Wedel, M. & 2 & Estados Unidos \\
\hline
\end{tabular}

Fonte: elaborada pelos autores.

Entre os autores que publicaram dois trabalhos, a maioria deles são dos Estados Unidos, país que lidera o ranking das publicações com 29 trabalhos, seguido da Austrália, com 10 estudos e da China, com 8 artigos. Na sequência, aparece a Alemanha $(\mathrm{N}=7)$, Países Baixos $(\mathrm{N}=6)$, Dinamarca $(\mathrm{N}=5)$, França $(\mathrm{N}=5)$, Canadá $(\mathrm{N}=4)$ e Inglaterra $(\mathrm{N}=4)$. Os países com 3 trabalhos publicados são: República Theca, Itália, Suécia e Taiwan. Além dos países citados, outros 25 são responsáveis por um ou dois trabalhos. Nota-se um predomínio norte-americano 
nas publicações sobre o tema, correspondente a 38,67\% do total, embora que países de diferentes continentes também apresentem estudos.

Analisando as fontes das publicações, há uma distribuição homogênea entre os periódicos, com destaque ao Journal of Marketing Research e ao Journal of Retailing and Consumer Services que publicaram 4 trabalhos sobre o tema, indicando que pesquisadores da área de Marketing desenvolvem estudos utilizando o eye tracking para compreender a tomada de decisão do consumidor. Já os periódicos que apresentam 3 publicações são: European Journal of Marketing, Journal of Business Research, Journal of Food Products Marketing e Organizational Behavior and Human Decision Processes. Para fins de análise, optou-se por considerar as fontes com três publicações ou mais, sendo que 55 fontes possuem um ou dois trabalhos publicados.

\subsection{Análise das estratégias de pesquisa}

As estratégias de pesquisa analisadas dizem respeito aos 18 trabalhos selecionados de acordo com os parâmetros do índice $h$. Sendo assim essas estratégias se referem à (i) natureza da pesquisa, (ii) tipo de pesquisa, (iii) delineamento da pesquisa, (iv) coleta e análise dos dados e (v) temática da pesquisa. Ao analisar os trabalhos publicados acerca da natureza da pesquisa, observa-se, de acordo com a Tabela 3, a predominância de estudos quantitativos, ou seja, $55,55 \%$ utilizaram essa estratégia metodológica, esse resultado pode estar associado aos dados fornecidos por meio da técnica de eye tracking, que permitem análises quantitativas a partir de suas métricas $\mathrm{O}$ método de triangulação (combinação de enfoques) foi adotado em 5 trabalhos, representando $27,28 \%$ dos artigos, e a pesquisa qualitativa teve menor incidência $(16,67 \%)$.

Tabela 3 - Natureza e Tipo de Pesquisa

\begin{tabular}{l|c|c|c|c|c}
\hline $\begin{array}{c}\text { Natureza da } \\
\text { pesquisa }\end{array}$ & $\begin{array}{c}\text { Total de } \\
\text { trabalhos }\end{array}$ & \% do total & Tipo de pesquisa & $\begin{array}{c}\text { Total de } \\
\text { trabalhos }\end{array}$ & \% do total \\
\hline Quantitativa & 10 & 55,5 & Explicativa & 14 & 77,8 \\
\hline Qualitativa & 3 & 16,7 & Descritiva / Explicativa & 1 & 5,6 \\
\hline Quali-Quanti & 5 & 27,8 & Teórica & 3 & 16,7 \\
\hline Total & $\mathbf{1 8}$ & $\mathbf{1 0 0}$ & Total & $\mathbf{1 8}$ & $\mathbf{1 0 0}$ \\
\hline
\end{tabular}

Fonte: elaborada pelos autores.

Em relação ao tipo de pesquisa (Tabela 3), nota-se o predomínio de estudos explicativos, correspondentes a 77,78\% dos trabalhos. De acordo com Sampieri, Collado e Lucio (2013), o 
principal interesse desse tipo de pesquisa é explicar por que um fenômeno ocorre e em que condições ele se manifesta, ou por que duas ou mais variáveis estão relacionadas.

Quanto ao delineamento da pesquisa, destaque-se a abordagem experimental, presente em 16 trabalhos $(88,89 \%)$, seguido de um trabalho de revisão de literatura $(5,56 \%)$ e um de revisão narrativa $(5,56 \%)$. Tal resultado corrobora com os achados sobre o tipo de pesquisa, no qual a ênfase explicativa se destacou. Dessa forma, evidencia-se que o uso do eye tracking nos estudos de tomada de decisão ocorre, na maioria, por meio de pesquisas explicativas e condução de experimentos.

Tendo em vista a existência de diferentes equipamentos para registrar o rastreamento ocular, observou-se que 14 estudos indicaram a ferramenta utilizada, enquanto 4 não informaram, sendo estes artigos caracterizados como descritivos ou teóricos. Entre os trabalhos que identificaram as ferramentas ou sistemas utilizados, destaca-se o rastreador ocular Tobii, contemplado em 7 estudos a partir de diferentes versões, Tobii 1750, Tobii Technology X60, Tobii Studio 1.3, Tobii T60XL e Tobii X1 Light, inclusive, alguns trabalhos optaram pelo uso de duas versões. Outros 3 estudos salientaram a utilização do SMI Eye-Link II e os demais selecionaram ferramentas como Eyegaze Binocular System, SensoMotoric Instruments RED-m, EyeTech ${ }^{\mathrm{TM}}$ e non-intrusive eye tracking.

$\mathrm{Na}$ Tabela 4, estão apresentadas as estratégias de coleta de dados utilizadas pelos trabalhos. Praticamente todos os artigos, com exceção de 2, abordaram as métricas geradas pelo eye tracking, especialmente, a coleta do número e da duração de fixações, uma das técnicas mais conhecidas e básicas para análise do rastreamento ocular e do processamento cognitivo (RAYNER, 1998; BARRETO, 2013).

O questionário foi utilizado como instrumento de coleta em 6 artigos, complementando as métricas do rastreador ocular em alguns casos. A análise documental foi empregada em 2 artigos teóricos. Além disso, um trabalho considerou os dados da atividade eletrodérmica dos participantes, um utilizou protocolo verbal e um empregou a revisão da literatura. 
Tabela 4 - Coleta de Dados

\begin{tabular}{l|c|c}
\hline Coleta de dados & Total de trabalhos & \% do total \\
\hline Métricas do eye tracking & 16 & 59,3 \\
\hline Questionário & 6 & 22,2 \\
\hline Análise documental & 2 & 7,4 \\
\hline Atividade eletrodérmica & 1 & 3,7 \\
\hline Protocolo verbal & 1 & 3,7 \\
\hline Revisão da literatura & 1 & 3,7 \\
\hline Total & $27 *$ & 100
\end{tabular}

*Os métodos de coleta podem ser múltiplos, superior ao total de artigos.

Fonte: elaborada pelos autores.

Conforme a análise dos dados mostrada na Tabela 5, a metade dos artigos analisou as métricas proporcionadas pela técnica eye tracking, como o número e a duração das fixações. Além dessas métricas, os artigos empregaram testes estatísticos univariados $(\mathrm{N}=17)$, bivariados $(\mathrm{N}=6)$ e multivariados $(\mathrm{N}=11)$. Um trabalho utilizou estatística não-paramétrica, um compreendeu análise visual e três valeram-se de técnicas de análise qualitativa. Destaca-se ainda que vários artigos combinaram diferentes estratégias de análise, totalizando 39 técnicas utilizadas.

Tabela 5 - Análise dos dados

\begin{tabular}{|c|c|c|c|}
\hline & Análise dos dados & $\mathbf{N}^{\circ}$ trabalhos & Total \\
\hline \multirow{3}{*}{ Estatística Univariada } & Métricas do eye tracking & 9 & \multirow{3}{*}{17} \\
\hline & Estatística descritiva & 7 & \\
\hline & Distribuição de Poisson & 1 & \\
\hline \multirow{2}{*}{ Estatística Bivariada } & Análise de Variância - ANOVA & 4 & \multirow{2}{*}{6} \\
\hline & Teste $\mathrm{t}$ & 2 & \\
\hline \multirow{5}{*}{ Estatística Multivariada } & Análise de Regressão & 6 & \multirow{5}{*}{11} \\
\hline & Análise de Covariância - ANCOVA & 1 & \\
\hline & Correlação & 2 & \\
\hline & Probabilidade log marginal & 1 & \\
\hline & Cadeia de Markov & 1 & \\
\hline Estatística não paramétrica & Teste U de Mann-Whitney & 1 & 1 \\
\hline Análise visual & Análises gráficas & 1 & 1 \\
\hline \multirow{3}{*}{ Análise qualitativa } & Análise de conteúdo & 2 & \multirow{2}{*}{3} \\
\hline & Análise de codificação & 1 & \\
\hline & & Total & 39* \\
\hline
\end{tabular}

*Os métodos de análise podem ser múltiplos, superior ao total de artigos.

Fonte: elaborada pelos autores.

Por fim, as temáticas de pesquisa dos 18 artigos são apresentadas (Quadro 1), sendo que os trabalhos com temas semelhantes foram agrupados para facilitar a compreensão. Observa-se que a atenção visual, o design visual e os efeitos da localização das informações são temas que têm despertado o interesse da comunidade acadêmica, que busca examinar o processamento das informações do indivíduo por meio dos movimentos de seus olhos. 
Quadro 1 - Temáticas de pesquisa

\begin{tabular}{|c|c|}
\hline Temáticas de pesquisa & $\begin{array}{c}\text { Contextualização das pesquisas } \\
\end{array}$ \\
\hline \multirow[t]{2}{*}{ Processo de escolha conjunta } & $\begin{array}{l}\text { O comportamento de aquisição de informações na análise conjunta } \\
\text { baseada em escolha }\end{array}$ \\
\hline & Processo de escolha conjunta e atenção na escolha \\
\hline \multirow{3}{*}{$\begin{array}{l}\text { Design visual e efeitos da } \\
\text { localização das informações }\end{array}$} & $\begin{array}{l}\text { Efeitos da localização na pesquisa visual e processos de tomada de } \\
\text { decisão }\end{array}$ \\
\hline & Exibições horizontais versus verticais de alternativas \\
\hline & $\begin{array}{l}\text { A influência do design visual nas reações dos consumidores às } \\
\text { classificações online }\end{array}$ \\
\hline Ferramentas neurofisiológicas & $\begin{array}{l}\text { Papel de ferramentas neurofisiológicas e de neuroimagem na } \\
\text { pesquisa de sistemas de informação }\end{array}$ \\
\hline Valor social & Orientação para o valor social \\
\hline Influência da marca & $\begin{array}{l}\text { Influência da marca em propagandas televisionadas e evasão } \\
\text { comercial }\end{array}$ \\
\hline \multirow{2}{*}{$\begin{array}{l}\text { Estratégias de escolha de } \\
\text { produtos }\end{array}$} & Busca de informações e estratégias de escolha de produtos online \\
\hline & Escolha de produtos em prateleiras \\
\hline \multirow{3}{*}{ Atenção visual } & $\begin{array}{l}\text { Medidas visuais de atenção ao produto e às informações e } \\
\text { sinalizações de exibição de preços }\end{array}$ \\
\hline & A influência do objetivo de compra na atenção visual de um cliente \\
\hline & Atenção ao preço em comércio eletrônico \\
\hline \multirow[b]{2}{*}{ Emoções na compra online } & Humor e impulsividade no comércio eletrônico \\
\hline & $\begin{array}{l}\text { As influências das respostas cognitivas e emocionais nas decisões de } \\
\text { compra }\end{array}$ \\
\hline Uso de heurísticas & $\begin{array}{l}\text { O uso da heurística Pwin em decisões que envolvem apostas mistas } \\
\text { complexas }\end{array}$ \\
\hline Recrutamento online & Sites de recrutamento online \\
\hline Ocultação de informações & Identificação de informações intencionalmente ocultas \\
\hline
\end{tabular}

Fonte: elaborada pelos autores.

Ademais, conforme pode ser observado no Quadro 1, as temáticas na sua maioria estão associadas à área de marketing, ratificando os demais resultados evidenciados anteriormente. 


\section{Considerações finais}

Este trabalho teve por objetivo investigar a produção de estudos que envolvem o uso de eye tracking em pesquisas sobre tomada de decisão, por meio de uma análise bibliométrica de trabalhos publicados na Web of Science, no período de 2009 a 2019. Para tanto, foram analisados 75 artigos que destacaram os principais aspectos que contemplam a evolução dos estudos.

Quanto aos autores que publicaram sobre o assunto, observou-se uma distribuição homogênea entre o número de publicações, uma vez que os pesquisadores publicaram um ou dois artigos, sendo desses, a maioria, oriundos dos Estados Unidos, Austrália e China. Ainda, sobre as fontes das publicações, notou-se uma distribuição análoga entre os periódicos, com destaque ao Journal of Marketing Research e ao Journal of Retailing and Consumer Services.

De acordo com a divisão de categorias proposta pela WOS, negócios e gestão lideram o ranking das publicações, isso se faz devido ao filtro utilizado nesta pesquisa (Business, Business Finance e Management) referente ao objetivo proposto. Por outro lado, ao ponderar as áreas de pesquisa, todos os trabalhos estão vinculados à área de Economia e Negócios e alguns artigos estão associados a outras áreas, como Psicologia, Ciência da Computação, Comunicação, Ciência da Informação e Biblioteconomia. Esses achados relacionam-se com o que Zamani, Abas e Amin (2016) salientam, ao considerar o campo de estudo do comportamento dos indivíduos e o rastreamento ocular multidisciplinares, conectando Eletrônica, Psicologia e Cognição. Com isso, verifica-se a abrangência do campo de estudo, sendo possível a produção, análises e comparações nas mais diferentes áreas.

Ao analisar os anos de publicação, 2018 foi o período que apresentou o maior número de trabalhos. Nesse sentido, compreende-se que o uso do eye tracking em pesquisas sobre tomada de decisão ainda é recente e está em desenvolvimento, já que no ano seguinte (2019) também se observou um volume semelhante de publicações. Desse modo, novos trabalhos que abordem o assunto podem se tornar importantes fontes de pesquisa, pois espera-se que apresentem os aprimoramentos do uso da técnica, bem como a sua aplicação em pesquisas envolvendo a tomada de decisão, contribuindo consequentemente com a teoria e com a dimensão prática sobre essa temática. 
No que diz respeito às estratégias de pesquisa utilizadas nos artigos investigados, podese constatar que a maioria corresponde aos estudos quantitativos, explicativos, fazendo uso da abordagem experimental. Quanto a coleta de dados, a maioria abordou as métricas geradas pelo eye tracking, especialmente, a coleta do número e da duração de fixações, utilizando principalmente a ferramenta de rastreamento ocular Tobii com diferentes versões. Além dessas métricas, os artigos empregaram testes estatísticos univariados, bivariados e multivariados, sendo que vários deles combinaram diferentes estratégias de análise. Por fim, em referência às temáticas desses estudos, os resultados evidenciaram que a atenção visual, o design visual e os efeitos da localização das informações são temas que têm despertado o interesse da comunidade acadêmica que está buscando examinar o processamento das informações do indivíduo por meio do rastreamento ocular.

Em síntese, essa pesquisa possibilitou compreender as características da produção científica internacional ao longo dos anos analisados sobre o uso de eye tracking em pesquisas sobre tomada de decisão. Com isso, demonstrou-se um panorama dos artigos publicados, seus autores, áreas, categorias, estratégias de pesquisa e demais aspectos, importantes para seu entendimento por parte dos leitores e demais pesquisadores interessados sobre o assunto.

Não obstante as suas contribuições, essa pesquisa limita-se ao apresentar à análise das publicações exclusivamente da base de dados Web of Science. Dessa forma, sugere-se para pesquisas futuras, que incluam novas fontes de coleta de dados, outras categorias de análise e ampliem o período de investigação. Além disso, podem ser utilizadas outras formas de apresentação dos resultados, com o uso de softwares, como VOSViewer, CitNetExplorer ou RStudio. Essas sugestões podem suprir as limitações desse estudo e instigar novas pesquisas que contribuam com o avanço teórico e prático sobre essa temática. 


\section{Referências}

BARRETO, A. M. S. B. Does brand's participation on Facebook affect positively its brandy equity? 2013. 326 p. Tese (Doutorado em Ciências da Comunicação) -Faculdade de Ciências Sociais e Humanas, Universidade Nova de Lisboa, Lisboa, 2013.

BAZERMAN, M. H.; MOORE, D. Processo decisório. Rio de Janeiro: Elsevier, 2010.

BENDER, C. S. O processamento da informação online e a decisão de compra em social commerce. 2019. 217f. Dissertação (Mestrado em Administração) - Universidade Federal de Santa Maria, Santa Maria, 2019.

BUETTNER, R. Investigation of the relationship between visual website complexity and users "mental workload: a neuro-is perspective: information systems and neuroscience. Springer International Publishing, Switzerland, p. 123-128, 2015. DOI 10.1007/978-3-31918702-0_16. Acesso em: 26 fev. 2020.

CASALINHO, G. A. O. When data changes pre-purchase behavior: the effects of information visualization on online information seeking. 2016. 160 p. Tese (Doutorado em Administração) - Universidade Federal do Rio Grande do Sul, Porto Alegre, 2016.

CHAE, S. W; LEE, K. C. Exploring the effect of the human brand on consumers' decision quality in online shopping: An eye-tracking approach. Online Information Review, United Kingdom, v. 37, n. 1, p. 83-100, 2013. DOI 10.1108/14684521311311649. Acesso em: 26 fev. 2020.

EDWARDS, W. The theory of decision making. Psychological Bulletin, Washington, v. 51, n. 4, p. 380-417, 1954.

GLYNN, P. D. et al. From data to decisions: processing information, biases, and beliefs for improved management of natural resources and environments. Earth's Future, [s. l.], v. 5, p. 356-378, 2017. DOI https://doi.org/10.1002/2016EF000487. Acesso em: 28 fev. 2020.

GOMES, L. F. A. M. Teoria da decisão. São Paulo: Thomson Learning, 2007.

HESS, E. H. Attitude and pupil size. Scientific American, v. 212, n. 4, p. 46-55, 1965.

HOLMQVIST, K. et al. Eye tracking: a comprehensive guide to methods and measures. Reino Unido: Oxford University Press, 2011.

JUST, M. A.; CARPENTER, P. A. A theory of reading: from eye fixations to comprehension. Psychological Review, Washington, v. 87, p. 329-354, 1980. DOI https://doi.org/10.1037/0033-295X.87.4.329. Acesso em: 28 fev. 2020.

MEIßNER, M.; MUSALEM, A.; HUBER, J. Eye tracking reveals processes that enable conjoint choices to become increasingly efficient with practice. Journal of Marketing Research, [s. l.], v. 53, p. 1-17, 2016. DOI https://doi.org/10.1509/jmr.13.0467. Acesso em: 27 fev. 2020. 
MEIßNER, M.; DECKER, R. Eye-tracking information processing in choice-based conjoint analysis. International Journal of Market Research, [s. l.], v. 52, n. 5, p. 593-612, 2010. https://doi.org/10.2501/S147078531020151X. Acesso em: 29 fev. 2020.

MELCHER, C. Proposta metodológica para avaliações otimizadas de usabilidade em websites desenvolvidos com método ágil: um estudo de caso. 2012. 299 f. Dissertação (Mestrado em Design) - Pontifícia Universidade Católica do Rio de Janeiro, Rio de Janeiro, 2012.

PEREIRA, B. A. D; LÖBLER, M. L.; SIMONETTO, E. O. Análise dos modelos de tomada de decisão sob o enfoque cognitivo. Revista de Administração da Universidade de Santa Maria, Santa Maria, v. 3, n. 2, p. 260-268, 2010. Disponível em: https://www.redalyc.org/articulo.oa?id=273420396008. Acesso em: 29 fev. 2020.

PÉREZ, I. J.; CABRERIZO, F. J.; HERRERA-VIEDMA, E. A mobile decision support system for dynamic group decision-making problems. IEEE Transactions On Systems, Man and Cybernetics, Canada, v. 40, n. 6, 2010. DOI 10.1109/TSMCA.2010.2046732. Acesso em: 29 fev. 2020.

PIMENTA, A. A. et al. A bibliometria nas pesquisas acadêmicas. Scientia, v. 4, n. 7, p. 1, 2017. Disponível em: https://docplayer.com.br/137842425-A-bibliometria-nas-pesquisasacademicas.html. Acesso em: 29 fev. 2020.

RAYNER, K. Eye movements in reading and information processing: 20 years of research. Psychological Bulletin, Washington, v. 124, n. 3, p. 372-422, 1998. DOI https://doi.org/10.1037/0033-2909.124.3.372. Acesso em: 29 fev. 2020.

RIEDL, R.; DAVIS, F. D.; HEVNER, A. R. Towards a neuro-is research methodology: intensifying the discussion on methods, tools, and measurement. Journal of the Association for Information Systems, Waco, v. 15, special issue, 2014. DOI 10.17705/1jais.00377. Acesso em: 29 fev. 2020.

RODAS, C. M.; MARCOS, M. C.; VIDOTTI, S. A. B. G. Tecnologia de eye tracking em user experience. In: ENCONTRO NACIONAL DE GESTÃO, POLÍTICAS \& TECNOLOGIAS DA INFORMAÇÃO. 2014, Goiás. Anais... Goiás: UFG, 2014.

ROULLET, B.; DROULERS, O. Neuromarketing: le marketing revisité par les neuroscien ces du consommateur. Paris: Dunod. 2010.

SAMPIERI, R. H.; COLLADO, C. F.; LUCIO, P. B. Metodologia de pesquisa. 5. ed. Porto Alegre: Penso, 2013.

SIMON, H. A. A behavioral model of rational choice. Quarterly Journal of Economics, United Kingdom, v. 69, p. 99-118, 1955.

VELHO, A. M. et al. S-Commerce: um estudo na perspectiva da Neuro-IS. In: ENCONTRO NACIONAL DA ASSOCIAÇÃO NACIONAL DE PÓS-GRADUAÇÃO E PESQUISA EM 
ADMINISTRAÇÃO - EnANPAD, 40., 2016, Rio de Janeiro. Anais... Costa do Sauípe: ANPAD, 2016.

WEB OF SCIENCE. Relatório de citações. 2020. Disponível em: http://appswebofknowledge.ez372.periodicos.capes.gov.br/WOS_GeneralSearch_input.do?product=WO

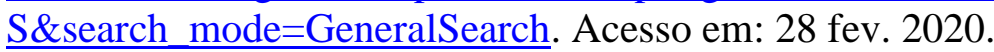

WEB OF SCIENCE. Eye tracking: análise de resultados. 2021. Disponível em: http://wcs.webofknowledge.com/RA/analyze.do?product=WOS\&SID=8AOEjo4M4nWuM5 AlZ8V\&field=PY_PublicationYear_PublicationYear_en\&yearSort=true. Acesso em: 15 fev. 2021.

WOLFRAM, D. Bibliometrics research in the era of big data: challenges and opportunities. In: BIBLIOMETRIA e cientometria no Brasil: infraestrutura para avaliação da pesquisa científica na era do Big Data, p. 91-101, 2017.

YARBUS, A. L. Eye movements and vision. New York: Plenum Press, 1967.

ZAMANI, H.; ABAS, A.; AMIN, M. K. M. Eye tracking application on emotion analysis for marketing strategy. Journal of Telecommunication, Electronic and Computer Engineering, [s. l.], v. 8, n. 11, p. 87-91, 2016. Disponível em:

https://journal.utem.edu.my/index.php/jtec/article/view/1415. Acesso em: 29 fev. 2020. 